\title{
Diagnóstico e recentes avanços no manuseio dos tumores do sulco superior do pulmão: revisão de literatura
}

Diagnosis and recent advances in the management of superior pulmonary sulcus tumors: a literature review

Wilson José Couto', Roberto Rivetti Suelotto ${ }^{2}$, Urias de Paula Filho³, José Renan Queiroz Guimarães ${ }^{4}$, Ana Lúcia Miguel Peres ${ }^{5}$

\section{Resumo}

Os tumores de sulco superior ou tumores de Pancoast são geralmente neoplasias pulmonares não-pequenas células que envolvem o ápice pulmonar. Devido à sua localização, apresentam características clínicas peculiares e dificuldades técnicas referentes ao tratamento cirúrgico.

Recentes avanços no manuseio dos pacientes com estes tumores incluem a terapia combinada com radioterapia e quimioterapia pré-operatórias, e novas abordagens cirúrgicas permitindo ressecções radicais e completas. Devido a isso, tem se observado uma melhoria dos resultados bem como do prognóstico dos pacientes portadores dessa condição clínica.

Palavras-Chave: Neoplasias pulmonares, Síndrome de Pancoast, Carcinoma pulmonar de células não-pequenas, Terapia combinada.

\section{Abstract}

Tumors of the superior pulmonary sulcus (Pancoast tumors) are usually non-small-cell lung cancers involving the pulmonary apex. Due to their location they are clinically unique and present limitations for surgical resection.

Recent advances in the management of such tumors include combined-modality therapy with preoperative concurrent chemotherapy and radiotherapy as well as new surgical approaches allowing radical and complete resection. This has improved the treatment outcomes and prognosis in patients with this clinical condition.

Key words: Pancoast syndrome, Non-small-cell lung carcinoma, Combined-modality therapy.

\footnotetext{
${ }^{1}$ Doutor em Medicina, Cirurgião Cardiotorácico - Hospital Samaritano - São Paulo.

${ }^{2}$ Cirurgião Cardiovascular - Hospital Samaritano - São Paulo.

${ }^{3}$ Oncologista Clínico - Hospital Samaritano - São Paulo.

${ }^{4}$ Oncologista Clínico - Hospital Samaritano - São Paulo.

${ }^{5}$ Hematologista e Oncologista Clínica - Hospital Metropolitano - São Paulo.

Trabalho realizado no Hospital Samaritano de São Paulo.

Endereço para correspondência: Dr Wilson José Couto - Rua Joaquim Távora 550, apt 131-B - Vila Mariana - 04015-011- São Paulo. E-mail: wilsoncouto10@yahoo.com.br
} 


\section{INTRODUÇÃO E DEFINIÇÃO}

Os tumores de sulco superior ou tumores de Pancoast constituem-se geralmente de câncer de pulmão nãopequenas células (CPNPC) que envolvem o ápice pulmonar. Recebem essa denominação pela sua relação com o sulco formado pela artéria subclávia no parênquima pulmonar do lobo superior. ${ }^{1,2}$

A definição de tumor de Pancoast clássica envolvia a presença de dor com irradiação distal para o braço, devido ao comprometimento das raízes inferiores do plexo braquial, além de manifestaçôes clínicas da síndrome de Horner, resultante do envolvimento do gânglio estrelado. ${ }^{1,3}$

Para ser considerado um tumor de Pancoast, é necessário ocorrer a invasão da pleura parietal em nível apical com sintomas de dor e/ou disfunção neurológica. Tais tumores podem invadir músculos, primeiros arcos costais, corpos vertebrais torácicos, vasos subclávios, porção inferior do plexo braquial e porção superior da cadeia torácica autonômica, incluindo o gânglio estrelado. $^{1,2}$ A síndrome de Pancoast é a constelação de sinais e sintomas que incluem dor no ombro e braço ao longo da distribuição do oitavo tronco nervoso cervical, primeiro e segundos troncos nervosos torácicos, síndrome de Horner, e fraqueza e atrofia da mão. Além do CPNPC, outros tipos de neoplasias e processos infecciosos podem também ocasionar a síndrome de Pancoast. ${ }^{3}$

\section{HISTÓRICO}

O primeiro relato de tumor envolvendo o ápice da cavidade torácica foi dado por Edwim Hare ${ }^{4}$. Entretanto coube a Henry Pancoast a descrição detalhada de 3 pacientes, que apresentavam tumores apicais cervicais, síndrome de Horner e atrofia da mão, relatados inicialmente em 1924, com atualização em 1932. ${ }^{67}$ Para distinguir esses casos das causas mais comuns de tumores apicais torácicos, Pancoast os denominou de "tumores do sulco superior".5

Desconhecido por Pancoast, Tobias, um médico argentino, fez ,em 1932, a mais importante descrição desses tumores. ${ }^{8}$ Tobias caracterizou precisamente sua anatomia, aspectos clínicos e também como sendo neoplasias pulmonares periféricas. A síndrome clínica associada aos tumores apicais torácicos é mais adequadamente referida como síndrome de Pancoast-Tobias, em homenagem às importantes contribuições de ambos os autores. ${ }^{5}$

O relato do primeiro caso com sobrevida tardia, após a ressecção em bloco do tumor e parede torácica, seguido de radioterapia adjuvante, foi feito por Chardack e Mac Callum, em 1956. ${ }^{9}$

A definição do tratamento moderno dos tumores de Pancoast foi feita por Shaw et al. ${ }^{10}$ Em sua casuística de
1961, 18 pacientes foram tratados com 3.500 cGy de radioterapia pré-operatória, seguida de ressecção cirúrgica do tumor com a parede torácica adjacente, vasos subclávios e plexo braquial, se necessário.

Desde os relatos de Shaw ${ }^{10}$, o manuseio dos tumores de Pancoast passou a ser considerado para o tratamento multimodal com protocolos de radioterapia, quimioterapia e cirurgia radical.

\section{QUADRO CLÍNICO}

Os principais sintomas associados aos tumores de Pancoast são em consequiência de seu comportamento localmente invasivo. ${ }^{1}$

A dor é o sintoma inicial mais comum e sua natureza depende das estruturas anatômicas envolvidas (arcos costais, corpos vertebrais, nervos e músculos da parede torácica etc). ${ }^{1}$

A manifestação dolorosa mais característica ocasionada pelos tumores de sulco superior é aquela ocasionada pela invasão do plexo braquial inferior. Ocorrem o envolvimento do ombro inferiormente e irradiação para a parte medial do braço (distribuição ulnar). Com a progressão da doença, pode se observar fraqueza do braço e atrofia dos músculos da parte medial do braço e mão.

Outra causa da dor no membro afetado é ocasionada pela distrofia simpática reflexa, resultante da destruição da porção superior do tronco simpático torácico. ${ }^{1}$

A invasão do gânglio estrelado (cervical superior) resulta na síndrome de Horner (ptose, miose e anidrose) no lado da face afetada. Além disso, a progressão do tumor pode levar à invasão do forame intervertebral com o subseqüente desenvolvimento de dor, disfunção neurológica, paraplegia e até quadriplegia, se não for tratada rapidamente. ${ }^{1}$

Nos tumores de Pancoast, o intervalo entre o início dos sintomas e o diagnóstico é geralmente prolongado (um ano ou mais). Isto se deve ao fato de os sintomas iniciais não serem de origem pulmonar. ${ }^{1}$

\section{DIAGNÓSTICO}

A radiografia simples de tórax demonstra um tumor apical, apesar de que, em alguns casos, a radiografia pode ser negativa e o tumor identificado apenas na tomografia axial computadorizada (TAC) do tórax. ${ }^{3}$ A TAC do tórax proporciona informação sobre a extensão do tumor de sulco superior, além de identificar a presença de outros nódulos pulmonares ou linfonodomegalia mediastinal. ${ }^{3}$

Nos tumores do sulco superior, a ressonância nuclear 
magnética (RNM) proporciona uma melhor avaliação da invasão tumoral através da pleura e gordura subpleural, do envolvimento do plexo braquial e dos vasos subclávios. ${ }^{3}$ Os cortes finos da RNM, na projeção coronal e sagital, têm uma acurácia maior do que a TAC de tórax (94\% versus $63 \%$ ) ao avaliar a invasão tumoral no sulco superior. ${ }^{11}$ Mais recentemente, a angioressonância nuclear magnética foi incorporada ao arsenal radiológico na avaliação dos tumores de Pancoast e parece ser superior na detecção do envolvimento vascular quando comparada com a RNM convencional e TAC de tórax. ${ }^{3}$

O papel da tomografia por emissão de pósitrons PET scan nesses tumores não está bem definido, mas tem sido utilizado no estadiamento do CPNPC. É útil na detecção de metástases linfonodais (com sensibilidade ao redor de $79 \%$ e especificidade de $91 \%$ ) e à distancia. ${ }^{3,12}$

Devido à localização periférica dos tumores de Pancoast, a broncoscopia e citologia são efetivas em estabelecer o diagnóstico em apenas 10 a 20\% dos casos ${ }^{12}$. O método diagnóstico mais comum para os tumores de sulco superior é a punção transtorácica percutânea guiada por ultra-sonografia ou tomografia. ${ }^{3,12}$

\section{ESTADIAMENTO}

Os tumores de sulco superior são estadiados como T3 ou T4, N0 a N3. Os tumores T3 são tumores de qualquer tamanho que invadem diretamente a parede torácica, diafragma, pleura mediastinal ou pericárdio. Os tumores T4 são aqueles que invadem o mediastino, coração, grandes vasos, traquéia, esôfago, corpo vertebral ou a carina. $\mathrm{O}$ envolvimento mediastinal linfonodal ipsilateral é considerado N2, enquanto o contralateral e supraclavicular N3. ${ }^{3}$

A sobrevida, em cinco anos, dos pacientes com tumor de Pancoast N2/N3 patológico é menor do que $10 \%$. Diante desse fato, justifica-se a avaliação mediastinal por meio de mediastinoscopia, ou talvez PET scan, com a finalidade de detecção de doença linfonodal não suspeita ${ }^{2}$. $\mathrm{Na}$ maioria das instituiçōes, os pacientes com doença linfonodal N2 comprovada por biópsia são considerados irresecáveis. ${ }^{5}$

O envolvimento supraclavicular ipsilateral é classificado como doença N3. Entretanto existe alguma evidência limitada de que os pacientes com tal comprometimento linfonodal podem apresentar sobrevida tardia aceitável. Devido a isso, as metástases linfonodais supraclaviculares ipsilaterais seriam mais adequadamente estagiadas como $\mathrm{N} 1$ em termos de prognóstico. $^{2}$

Com o advento de abordagens terapêuticas mais agressivas para os tumores de Pancoast, tornou-se mandatório um estadiamento extratorácico extenso através de TAC de abdômen superior, RNM de sistema nervoso central, cintilografia óssea ou PET scan. Tal investigação deve ser realizada mesmo em pacientes sem sintomas sugestivos de metástases sistêmicas. ${ }^{2}$

\section{TRATAMENTO}

\section{CiRuRgIA}

A ressecção de um tumor de Pancoast idealmente envolve a realização de lobectomia com ressecção em bloco da área da parede torácica envolvida.

Nos pacientes submetidos à ressecção completa, a lobectomia proporciona uma melhor sobrevida quando comparada com a ressecção em cunha com sobrevida em cinco anos de $60 \%$ versus $33 \%^{2}$. A sobrevida dos pacientes após a ressecção incompleta é semelhante à de não ressecção, enfatizando a importância do tratamento cirúrgico radical. ${ }^{2}$

\section{TÉCNICAS OPERATÓRIAS}

A técnica clássica foi popularizada por Paulson e Shaw ${ }^{10}$ e consiste em toracotomia póstero-lateral alta, com divisão dos músculos entre a escápula e os processos espinhosos e elevação da escápula para fora da cavidade torácica para expor os arcos costais posteriormente. Esta abordagem permite excelente exposição da parede torácica posterior, incluindo os processos transversos, as raízes nervosas torácicas e o hilo pulmonar. ${ }^{1,2}$

Tatsumura ${ }^{13}$ descreveu uma variação técnica, com a incisão se estendendo da linha axilar anterior obliquamente até a fúrcula supra-esternal. A vantagem de tal acesso seria facilitar a abordagem da região torácica ântero-superior em caso de necessidade de ressecção e reconstrução vascular.

Para os tumores de Pancoast com componente anterior importante e envolvimento dos vasos subclávios, a melhor abordagem é através do acesso anterior, transclavicular, descrito por Dartevelle ${ }^{14}$. Nessa técnica, é realizada uma incisão ao longo da borda anterior do músculo esternocleidomastoídeo que continua lateralmente sobre a clavícula. O músculo é dividido e a metade medial da clavícula é ressecada. ${ }^{1,2}$

Grunenwald e Spaggiani ${ }^{15}$ descreveram a técnica da cervicotomia com esternotomia transmanubrial superior e incisão subclavicular, com preservação da clavícula, da mobilidade do ombro, e sem danos estéticos. A técnica de Masaoka ${ }^{16}$ utiliza cérvico-esterno toracotomia e é outra opção para os tumores apicais anteriores e com envolvimento vascular. A incisão do tipo hemiclamshell ("trap door") é uma modificação de Masaoka, 
em que a porção cervical do procedimento é omitida. ${ }^{1}$ Para a ressecção de tumores mais extensos, pode ser necessária a combinação das várias formas de abordagem descritas acima. ${ }^{2}$

O valor da ressecção cirúrgica dos tumores de sulco superior com invasão de corpo vertebral é controverso, mas o avanço das técnicas operatórias permitiu ressecçôes extensas, que devem ser realizadas em centros especializados, onde é possível uma colaboração do cirurgião de coluna com o cirurgião torácico. ${ }^{2}$

\section{RADIOTERAPIA}

O tratamento radioterápico tem sido utilizado como terapia única ou combinada. A sobrevida em cinco anos dos pacientes tratados com radioterapia exclusiva pode chegar a $23 \% .{ }^{12}$ As técnicas de planejamento radioterápico modernas permitem uma distribuição mais adequada da dose a ser utilizada e a possibilidade de aumento gradual de dose sem acréscimo de morbidade. ${ }^{12}$ Formas alternativas de radioterapia têm sido utilizadas, associadas ou não à radioterapia externa convencional. A utilização de braquiterapia e radioterapia intraoperatória mostra resultados promissores em pacientes selecionados. ${ }^{12}$

Entretanto essas técnicas não estão facilmente disponíveis e não existem informações relacionadas a suas morbidades tardias. ${ }^{12}$

\section{QUIMIOTERAPIA}

O tratamento quimioterápico isolado é usado de forma paliativa, diante da evidência de doença metastática ou recorrência após o tratamento local.

Os esquemas utilizados são geralmente baseados em platinas, usados rotineiramente no tratamento do CPNPC. ${ }^{1}$

$\mathrm{O}$ benefício principal do tratamento quimioterápico é melhorar o controle de sintomas, principalmente a dor relacionada com o crescimento tumoral local (que é severa e causa prejuízo importante na qualidade de vida). ${ }^{1}$

\section{TRATAMENTO COMBINADO}

O tratamento clássico para os tumores de sulco superior consistia de radioterapia pré-operatória seguida de cirurgia, como descrito por Paulson. ${ }^{17}$ A vantagem potencial de tal abordagem seria o aumento da ressecabilidade, a diminuição da disseminação tumoral durante a cirurgia, o bloqueio de canais linfáticos (provavelmente evitando metástases) e a maximização do controle local. ${ }^{12}$

Mais recentemente, o uso de quimioterapia e radioterapia pré-operatórias tem sido avaliado e preconizado como o tratamento padrão.

$\mathrm{O}$ estudo norte-americano "intergroup trial 0160" é o primeiro estudo prospectivo multi institucional de CPNPC do sulco superior. Neste estudo fase dois, 111 pacientes com mediastinoscopia negativa, clinicamente T3-4, NO-1, foram submetidos à terapia de indução. ${ }^{5,18}$ O tratamento consistiu de dois ciclos de etoposide e cisplatina com 45 cGy de radioterapia concomitante. Os pacientes com doença estável ou regressão tumoral foram submetidos à toracotomia e ressecção pulmonar anatômica, seguida de dois ciclos adicionais de quimioterapia pós-operatória., ${ }^{5,18}$ Dos 111 pacientes iniciais, 83 (75\%) foram operados. A terapia de indução foi bem tolerada pela maioria dos pacientes e apenas 5 pacientes apresentaram toxicidade grau 3 ou maior. Proporcionou também significante esterilização do tumor primário. Um terço dos pacientes apresentou resposta completa ao tratamento confirmada histologicamente, e outro terço apresentou doença microscópica mínima na peça ressecada. Dentre os 60 pacientes que apresentavam tumores T3, 91\% foram submetidos à ressecção completa, bem como $87 \%$ dos T4. ${ }^{5,18}$

Os resultados mais consistentes dos estudos acima mostraram uma sobrevida em cinco anos de $41 \%$ para todos os pacientes e $53 \%$ após a ressecção completa. $\mathrm{O}$ grau de resposta ao tratamento, confirmada histologicamente, foi fator preditivo de sobrevida. A taxa de recorrência local foi de 12\% (melhor quando comparadas com controles históricos de 40\%). ${ }^{5,19}$

Portanto a terapia de indução com quimioterapia e radioterapia foi bem tolerada e proporcionou um alto índice de esterilização tumoral, ressecção completa e controle local. ${ }^{5}$

O protocolo do "Japan Clinical Oncology Group" (JCOG) 9806 é similar ao estudo norte americano e com resultados semelhantes. ${ }^{20}$

Wright et al. ${ }^{21}$ do Massachusetts General Hospital revisaram os pacientes submetidos no pré-operatório à radioterapia com quimioterapia ou radioterapia isolada em sua instituição. Os pacientes do primeiro grupo apresentaram uma resposta completa ao tratamento maior ( $66 \%$ versus $13 \%$, confirmado histologicamente), além de menor índice de recorrência local e maior sobrevida ( $84 \%$ versus $49 \%$ em quatro anos).

Tendo em vista os resultados acima, o tratamento dos tumores de sulco superior com quimioterapia e radioterapia pré-operatoriamente representa uma excelente opção na abordagem de tumores de Pancoast, e seu uso pode ser considerado como o tratamento padrão. ${ }^{2,5}$

\section{Perspectivas futuras}

Os avanços nas técnicas operatórias para os tumores de Pancoast permitem a ressecção de vasos subclávios e 
até de corpos vertebrais, mas o envolvimento extenso do plexo braquial continua sendo uma contra-indicação para a ressecção. O tratamento paliativo ou com radioterapia exclusiva, algumas vezes, leva a um braço não funcional devido ao crescimento tumoral. Parece razoável explorar-se a possibilidade de ressecçóes cirúrgicas mais extensas como, por exemplo, a amputação de antebraço. É possível que a morbidade dessa operação extensa seja menor do que a morbidade ocasionada pelo crescimento tumoral, bem como proporcionaria uma possibilidade de cura. $^{2}$

Com a finalidade de diminuir-se a incidência de recorrências sistemicas à distância, novos estudos são necessários. O "Southwestern Oncology Group (SWOG)-0220 Trial" tem como objetivo investigar o uso de docetaxel no pós-operatório de pacientes já submetidos à quimioterapia e radioterapia antes da ressecção cirúrgica. ${ }^{5,12}$

A irradiação cranial profilática (ICP) pode ser um tratamento adicional para os tumores de sulco superior. Isto é baseado na observação de que o cérebro é o local mais comum de primeira recorrência e muitas vezes o único. Geralmente, a ICP é bem tolerada e pode ser administrada no pós-operatório, após a ressecção completa. ${ }^{2}$

\section{CONCLUSÕES}

O tratamento dos pacientes com tumores de Pancoast ainda continua a ser um desafio. Avanços foram feitos no conhecimento anatômico e biológico desses tumores, levando ao conseqüente desenvolvimento de novas modalidades terapêuticas. Incisōes cirúrgicas alternativas e novas técnicas permitem a ressecção de estruturas consideradas previamente irresecáveis. $\mathrm{O}$ tratamento quimioterápico, adicionado à abordagem tradicional de radioterapia pré-operatória, aparentemente melhorou o controle tumoral local, os índices de ressecção completa e a cura.

\section{REFERÊNCIAS}

1. Kraut MJ, Vallieres E, Thomas CR Jr. Pancoast (superior sulcus) neoplasms. Curr Probl Câncer. 2003;27:81-104.

2. Detterbeck FC. Changes in the treatment of Pancoast tumors. Ann Thorac Surg. 2003; 75:1990-7.

3. Jett JR. Superior sulcus tumors and Pancoast's syndrome. Lung Cancer. 2003;42 Suppl 2:S17-21.

4. Hare ES. Tumor involving certain nerves. Med Gaz. $1838 ; 1: 16-8$.

5. Kent MS, Bilsky MH, Rusch VW. Resection of superior sulcus tumors (posterior approach). Thorac Surg Clin. 2004;14:217-28.

6. Pancoast H. Importance of careful roentgem-x ray investigation of apical chest tumors. JAMA. 1924;83:1407-11.

7. Pancoast HK. Superior pulmonary sulcus tumor. JAMA. 1932;99:1391-6.

8. Tobias J. Sindrome apico-costo-vertebral doloroso for tumor apexiano: su valor diagnostico en el cancer primitivo pulmonary. Rev Med Lat Am. 1932;19:1552-6.

9. Chardack WM, MacCallum JD. Pancoast tumor: five-year survival without recurrence or metastases following radical resection and postoperative irradiation. J Thorac Surg. 1956;31:535-42.

10. Shaw RR, Paulson DL, Kee Jr JL. Treatment of the superior sulcus tumor by irradation followed by resection. Ann Surg. 1961;7:29- 40.

11. Heelan RT, Demas BE, Caravelli JF, Martini N, Bains MS, McCormack PM, et al. Superior sulcus tumors: CT and MR imaging. Radiology. 1989;170:637-41.

12. Archie VC, Thomas Jr CR. Superior sulcus tumors: a minireview. Oncologist. 2004; 9:550-5.

13. Tatsumura T, Sato H, Mori A, Miyazaki M, Koyama , Tsujimoto M, et al. A new surgical approach to apical segment lung diseases, including carcinomas and inflammatory diseases. J Thorac Cardiovasc Surg. 1994;107:32-6.

14. Dartevelle PG, Chapelier AR, Macchiarini P, Lenot B, Cerrina J, Ladurie FL, et al. Anterior transcervical-thoracic approach for radical resection of lung tumors invading the thoracic inlet. J Thorac Cardiovasc Surg. 1993; 105:1025-34.

15. Grunenwald D, Spaggiari L. Transmanubrial osteomuscular sparing approach for apical chest tumors. Ann Thorac Surg. 1997; 63:563-6.

16. Masaoka A, Ito Y, Yasumitsu T. Anterior approach for tumor of the superior sulcus. J Thorac Cardiovasc Surg. 1979; 78:413-5.

17. Paulson DL. The survival rate in superior sulcus tumors treated by presurgical irradiation. JAMA. 1966;196:342.

18. Rusch VW, Giroux DJ, Kraut MJ, Crowley J, Hazuka M, Johnson DH, et al. Induction chemoradiation and surgical resection for non-small cell lung carcinomas of the superior sulcus: Initial results of Southwest Oncology Group Trial 9416 (Intergroup Trial 0160). J Thorac Cardiovasc Surg. 2001; 121:472-83.

19. Rusch VW, Giroux DJ, Kraut MJ, Crowley J, Hazuka M, Johnson DH, et al. Induction chemoradiation and surgical resection for non-small cell lung carcinomas of the superior sulcus: prediction and impact of pathologic complete response. Lung Cancer. 2003; 41(suppl 2):s78.

20. Kunitoh H, Kato H, Tsuboi M, Ishizuka N, Tsuchiya R, Ichinose I, et al. A phase II trial of preoperative chemoradiotherapy followed by surgical resection in Pancoast tumors: Initial report of Japan Clinical Oncology Group trial (JCOG 9806). Proc Am Soc Clin Oncol. 2003; 22:2549a. 
21. Wright CD, Menard MT, Wain JC, Donahue DM, Grillo $\mathrm{HC}$, Lynch TJ, et al. Induction chemoradiation compared with induction radiation for lung cancer involving the superior sulcus. Ann Thorac Surg. 2002; 73: 1541-4. 